\title{
Impact of Initial Degree of Coronary Narrowing on Left Ventricular Function in First Myocardial Infarction
}

\author{
Shinichiro Yamagami, ${ }^{1} \mathrm{MD}$, Katsumi Miyauchi, ${ }^{1} \mathrm{MD}$, and Hiroyuki Daida, ${ }^{1} \mathrm{MD}$
}

\begin{abstract}
SUMMARY
To determine whether the initial degree of coronary narrowing prior to a first myocardial infarction (MI) affects left ventricular function, we analyzed paired coronary angiograms and left ventriculography in 41 consecutive MI patients in whom coronary angiography was obtained before and after MI.

Patients were divided into 2 groups according to coronary narrowing of the infarct culprit lesion at first angiogram; a significant narrowing group (group S, 15 patients) and a nonsignificant narrowing group (group N, 26 patients). Significant narrowing was defined as more than $75 \%$ stenosis of an infarct-related segment in the first angiography and nonsignificant narrowing was less than 50\% narrowing. Clinical characteristics were similar in the two groups, with the exception of initial diameter stenosis. LV function was normal in both groups at initial examination. Group S patients had a higher prevalence of angina prior to MI ( $73 \%$ versus $23 \% ; P=0.001)$, good collateral development (73\% versus 35\%; $P=0.02)$, and non-Q MI (73\% versus $27 \% ; P=0.004)$ than group N patients. RWM was also superior in group S compared with group $\mathrm{N}$. The deterioration of global and infarction zone function was mild in group S compared with group N (group S; median EF -10\%, RWM -0.27 SD/chord, group N; median EF -26\%, RWM -1.62 SD/chord, $P=0.001$ ).

We conclude that deterioration of LV wall motion in patients with severe stenosis in their initial stenosis would be milder than in AMI that developed from a mild degree of stenosis. (Int Heart J 2010; 51: 82-85)
\end{abstract}

Key words: Coronary narrowing, Left ventricular function, Myocardial infarction

$\mathrm{L}$ eft ventricular (LV) function is an important predictor of survival in patients with acute myocardial infarction (AMI). ${ }^{1)}$ Thus, the determination of $\mathrm{LV}$ function may be a critical issue as the consequence of coronary occlusion. In a retrospective study, the incidence of total occlusions from severe stenosis was three times higher than from less severe lesions. ${ }^{2)}$ However, this process only results in small or silent myocardial infarction or no infarction. ${ }^{3)}$ Thus, the initial degree of coronary narrowing before MI may be one of the factors that determine LV function. There is surprisingly little data assessing the initial degree of narrowing before AMI influences the LV function. The aim of the present study was to evaluate the relation between the severity of preinfarction narrowing of the infarctrelated artery and changes in the LV function after a first MI in patients without revascularization treatment.

\section{MeTHODS}

We evaluated the coronary angiograms (CAG) of 41 medically treated patients with coronary artery disease who had undergone CAG and left ventriculography (LVG) before and after MI. The second angiogram was performed to determine the location of the infarction producing obstruction and left ventricular function: global ejection fraction and infarction zone wall motion, and the first angiogram was analyzed to assess the coronary anatomy and wall motion preceding the MI.

Study patients: We reviewed the records of all patients who underwent CAG and LVG at Juntendo University hospital between December 1, 1990 and April 2000. To be included in this series the following criteria had to be fulfilled: (1) patients who underwent CAG and LVG before and after the onset of MI, (2) the first angiogram was undertaken to evaluate symptoms of angina pectoris or an abnormal exercise test, or both, at least 1 year before Ml. At this time, LV function was normal, (3) the second angiogram was performed within 1 month after the first MI and an infarctrelated artery (IRA) showed total or subtotal obstruction. The exclusion criteria were (1) history of previous MI, (2) subsequent coronary intervention (angioplasty or coronary bypass), (3) treatment by thrombolysis or angioplasty after AMI, (4) inadequate LVG was available and unsuitable for quantitative analysis, (5) additional cardiac abnormality patients (valvular heart disease, cardiomyopathy), and (6) patients without a clear clinical history. Thus, we selected patients who had normal LV function before AMI and had total or subtotal occlusion of the LAD or RCA in IRA after MI.

The diagnosis of MI was based on electrocardiography changes and rises in serum enzymes and confirmed by the

From the ${ }^{1}$ Department of Cardiology, Juntendo University School of Medicine, Tokyo, Japan.

Address for correspondence: Shinichiro Yamagami, MD, Department of Cardiology, Juntendo Tokyo Koto Geriatric Medical Center, 3-3-20 Shinsuna, Kotoku, Tokyo 136-0075, Japan.

Received for publication October 22, 2009.

Revised and accepted November 9, 2009. 
presence of new wall motion abnormalities on LVG. In this retrospective analysis, the first CAGs were reviewed and classified into 2 groups according to the coronary narrowing of the culprit lesion on the first angiogram; a significant narrowing group (group S, 15 patients) and a nonsignificant narrowing group (group N, 26 patients). Significant narrowing was defined as more than $75 \%$ stenosis of an infarct related segment in the first angiography and nonsignificant narrowing was defined as less than $50 \%$ narrowing.

Clinical evaluation: A series of parameters were coded, stored, and studied in a retrospective manner. They included age, sex coronary risk factor (history of diabetes mellitus, hypertension, hyperlipidemia and tobacco) and medications before AMI. CAG data were included: interval from first CAG to MI, the number of diseased vessels, location of IRA, \% stenosis at initial CAG of subsequent IRA, and degree of collateral circulation.

All coronary angiograms were reviewed by 2 independent observers who were blind to the subsequent clinical course of the patients. Selective coronary angiography was performed using multiple projections after nitroglycerin infusion. The degree of coronary stenosis was determined by quantitative coronary angiography using a Kontron Cardiac 500. LVG was performed in the right oblique projection. Global LV ejection fraction was estimated according to the area length method. The regional wall motion and percent length of abnormal contractile segment were calculated using the center line method using 100 chords. ${ }^{4)}$ Each shortening fraction was normalized by the end-diastolic perimeter of LV at end-systole. This normalized wall motion in the territory of the left anterior descending coronary artery (chord 10 to 66) and territory of the right coronary artery (chord 77 to 96) was expressed as the standard deviation from the mean regional wall motion value previously determined in 50 age-matched normal subjects.

Non-Q wave myocardial infarction: A typical history of chest pain with new and persistent ST and T wave changes on ECG, lasting $>48$ hours without the evolution of new $Q$ waves and without a new severe segmental wall motion abnormality on LVG.

Collateral vessels: Collateral vessels were scored according to the degree of opacification of the native vessel distal to its occlusion. Collateral vessels were assigned a numeric score between 0 and 3 according to the criteria of Cohen and Rentrop. ${ }^{5)}$ The classification system is summarized as follows: grade $0=$ no visible filling of any collateral channels; grade 1 = filling of side branches of the artery to the

Table I. Baseline Characteristics of Patients

\begin{tabular}{lccc}
\hline & Group S & Group N & $P$ \\
\hline Number of patients & 15 & 26 & \\
Age (years) & $52.5 \pm 1.9$ & $53.1 \pm 1.9$ & 0.83 \\
Male gender (\%) & 100 & 92 & 0.88 \\
Number of disease vessels & $1.7 \pm 0.7$ & $1.2 \pm 0.9$ & 0.42 \\
Interval from cath 1 to MI (Days) & $4710 \pm 437$ & $5304 \pm 382$ & 0.32 \\
\% stenosis at initial CAG & $81.2 \pm 4.1$ & $22.1 \pm 4.0$ & 0.0001 \\
$\quad$ of subsequent IRA & & & \\
Infarct-related artery & & & 0.66 \\
LAD & 9 & 15 & \\
RCA & 6 & 11 & \\
\hline
\end{tabular}

perfused via collateral vessels without visualization of the epicardial segment; grade $2=$ partial filling of the epicardial segment by collaterals; and grade $3=$ complete filling of the epicardial segment by collaterals. Good collaterals were defined as those above grade 3 .

Antecedent angina: Antecedent angina was defined as Canadian class 1 to IV angina occurring within 1 week before the onset of MI.

Statistical analysis: The data are expressed as proportions or as the mean value \pm standard deviation (SD). Intergroup differences were analyzed by the chi-square test or the Fisher exact test. Continuous variables were analyzed using the Student test. Two-tailed $P$ values were used for all statistical testing. For multivariate analysis, logistic regression analysis was used to determine the factors independently related to LV function. Statistical significance was defined as a $P<0.05$.

\section{RESULTS}

The clinical and angiographic characteristics of the patients are summarized in Tables I and II. All characteristics were similar in both groups, with the exception of the initial diameter stenosis of IRA. Concomitant medication before and after administration included nitroglycerin, beta blockers, and calcium channel blockers, and did not differ statistically between the groups. The indication for the first angiogram was chest pain consistent with angina in all patients. The second coronary angiogram was performed $18 \pm$ 4 days after MI. The interval from the initial catheterization until the development of MI was less in the group $\mathrm{S}$ patients (26.2 \pm 22.4 months) than in the group $\mathrm{N}$ patients $(40.4 \pm$ 27.4 months), but the difference was not statistically significant.

The incidence of prodromal angina was significantly higher in group $\mathrm{S}$ than in group $\mathrm{N}$ (73\% versus $23 \% ; P=$ 0.02) (Figure 1). Compared with the group $\mathrm{N}$ patients, group $\mathrm{S}$ patients had a higher frequency of good collaterals $(73 \%$ versus $35 \% ; P=0.05)$. The prevalence of non-Q wave MI was significantly higher in group $\mathrm{S}$ than in group $\mathrm{N}$ patients $(73 \%$ versus $27 \% ; P=0.05)$.

Comparison of global cardiac function between the 2 groups (Figure 2): Ejection fraction was normal and asynergy was not observed before infarction in either group. Global ejection fraction in group $\mathrm{N}$ decreased significantly from $64 \pm$

Table II. Previous Medications and Risk Factors

\begin{tabular}{lccc}
\hline & $\begin{array}{c}\text { Group S } \\
(n=15)\end{array}$ & $\begin{array}{c}\text { Group N } \\
(n=26)\end{array}$ & $P$ \\
\hline Previous medication & & & \\
$\quad$ Beta-blockers (\%) & 40.0 & 23.1 & 0.39 \\
Ca-channel blockers (\%) & 73.3 & 57.6 & 0.31 \\
$\quad$ Aspirin (\%) & 93.3 & 76.9 & 0.15 \\
Hypertension (\%) & 80.0 & 57.6 & 0.14 \\
Diabetes mellitus (\%) & 33.3 & 23.1 & 0.47 \\
History of smoking (\%) & 80.0 & 69.0 & 0.45 \\
Hyperlipidemia (T-cho $>220)$ & 66.6 & 57.7 & 0.56 \\
T-cho (mg/dL) & $237 \pm 11$ & $234 \pm 11$ & 0.69 \\
TG (mg/dL) & $203 \pm 19$ & $190 \pm 20$ & 0.61 \\
HDL (mg/dL) & $40 \pm 4$ & $47 \pm 5$ & 0.25 \\
\hline
\end{tabular}




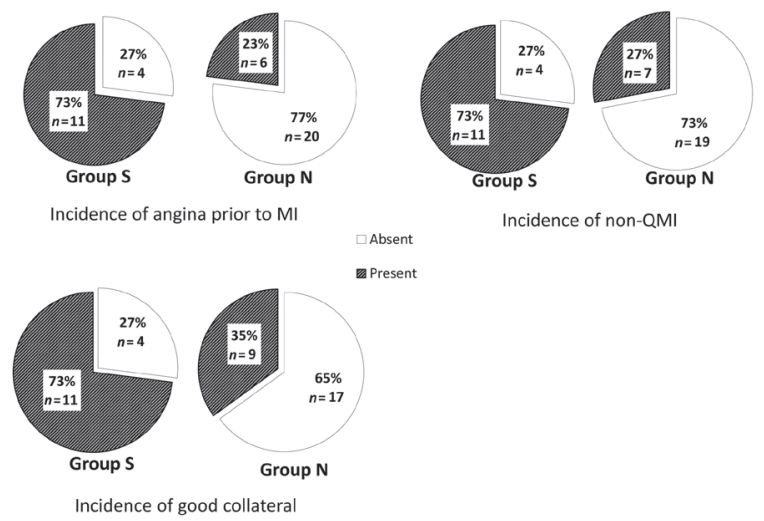

Figure 1. Graphs showing percentage of angina prior MI, good collateral, and non-Q MI in both groups. Group S: significant narrowing group (15 patients), Group N: nonsignificant narrowing group (26 patients). Significant narrowing was defined as more than $75 \%$ stenosis of the infarct-related segment in the first angiography and nonsignificant narrowing was defined as less than 50\% narrowing.

9 to $35 \pm 12 \%$. In contrast, no significant decrease in global ejection fraction was observed in group $\mathrm{S}$ (from $63 \pm 9$ to $53 \pm 12 \%)$. Group $\mathrm{S}$ had a higher mean global LVEF after MI than group N. Among the patients with an anterior wall infarction, those in group $\mathrm{N}$ had a significant decrease in global ejection fraction (from $66 \pm 8$ to $33 \pm 8 \%, P=0.01$ ), whereas those in group $\mathrm{S}$ had no significant reduction in global ejection fraction (from $64 \pm 12$ to $50 \pm 9 \%, P=0.10$ ). The deterioration of global function was mild in group $\mathrm{S}$ compared with group $\mathrm{N}$. Left ventricular ejection fraction in group $\mathrm{N}$ patients with inferior wall infarction decreased from $62 \pm 8$ to $40 \pm 8 \%(P=0.05)$ whereas ejection fraction in group $\mathrm{S}$ showed no significant serial change $(61 \pm 5$ to $56 \pm 6 \%, P=0.12$ ). The deterioration of global function was also mild in group $\mathrm{N}$ compared with group $\mathrm{S}$.

Regional wall motion (Figure 3): Regional wall motion (RWM) was also superior in group S compared with group $\mathrm{N}$. The deterioration of infarct zone function was mild in group S compared with group N (group S; RWM- 0.27 SD/ chord, group N; RWM- 1.62 SD/chord). Analysis of RWM showed that the superiority of global ejection fraction in group $\mathrm{S}$ was due to maintenance in the infarction area (from $12.1 \%$ to $11.9 \%$ in patients with anterior wall infarction and from $11.1 \%$ to $9.9 \%$ in patients with inferior wall infarction) and not to hyperkinesis of the noninfarcted area.

Variables contributing to recovery of left ventricular function: Multiple regression analysis was performed to evaluate the contribution of each variable to the cardiac function. Multivariable analysis identified only preinfarction severe stenosis as a significant predictor of a decrease in left ventricular ejection fraction and regional wall motion $(P<$ $0.0001)$.

\section{Discussion}

This study showed that the nonsignificant stenosis in an infarct-related lesion before MI was correlated with preserved LV function in patients with a first MI. We ob-

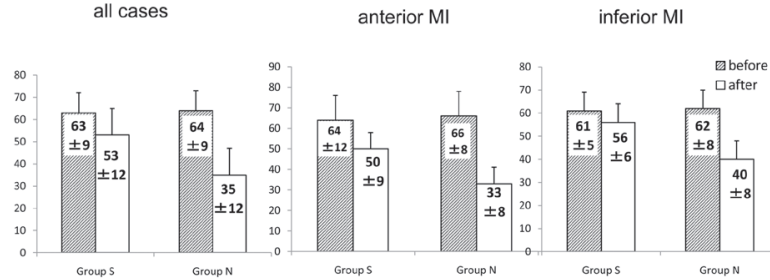

Figure 2. Left ventricular ejection fraction in patients with anterior and inferior myocardial infarction before and after infarction in both groups. Group S: significant narrowing group (15 patients), Group N: nonsignificant narrowing group (26 patients).

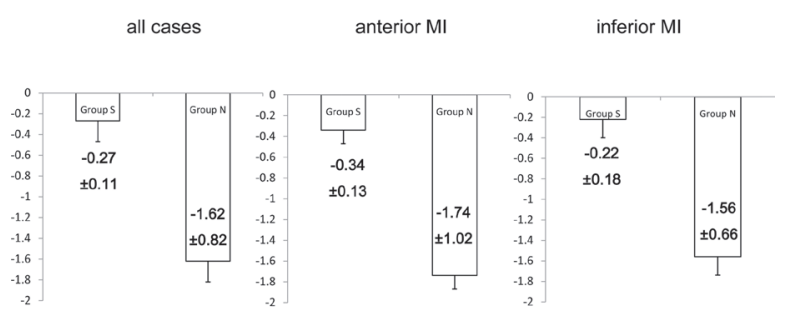

Figure 3. Deterioration of left ventricular regional wall motion after infarction in patients with an anterior myocardial infarction and in patients with an inferior myocardial infarction.

served a decrease in ejection fraction in the patients who had progression from less than 50\% narrowing to occlusion, whereas no significant change in LV function was found in the patients with progression from more than $90 \%$ narrowing to occlusion.

Several studies have examined the implications of preinfarct related stenosis on LV function after myocardial infarction. Ambrose, $e{ } \mathrm{al}^{2)}$ reported that severe preexisting stenosis prevented Q-wave MI. This study did not evaluate the effect of pre-existing stenosis on LV function. However, our study extended the advance effect of severe pre-existing stenosis on non-Q wave infarction to preserve LV function. Visser, $e \mathrm{al}^{3)}$ showed that deterioration of LV function from less than $90 \%$ in IRA was less than that from $50 \%$ stenosis. However, their study results were different from our findings with respect to the following points. In our study, all patients were first MI patients and LV function was initially normal. Their study included patients with a previous MI. Moreover, our target vessels were only the RCA and $\mathrm{LAD}$ but not the $\mathrm{CX}$ because the right anterior oblique projection, as used in our study and the Visser, et al study, is of limited value in showing contraction of the area supplied by the CX. In spite of these limitations, the CX was involved in 305 cases in their study. Our clinical study was the first study regarding the effect of preinfarct related vessel stenosis on LV function using paired CAG and LVG before and after MI in patients with their first MI.

Mechanisms of collateral development and preconditioning: Preservation of left ventricular function postinfarction is determined by several factors such as the presence or absence of antecedent angina pectoris ${ }^{6}{ }^{6}$ collateral circulation $;{ }^{7,8)}$ patency of the infarct-related artery;" residual coronary stenosis ${ }^{10)}$ time from onset of infarction to reperfusion; and 
post reperfusion therapy. ${ }^{11)}$ In our study, collateral circulation and antecedent angina pectoris were more likely to be present in group $\mathrm{S}$ than in group $\mathrm{N}$. These conditions explained the salvage effect of pre-existing severe stenosis on LV function. A well-developed collateral channel prohibited myocardial ischemia or transmural necrosis. The most plausible explanation for good collateral development and antecedent angina was the long-standing recurrent ischemia due to the high degree of stenosis, whereas abrupt closure of moderate stenosis would not leave time for the collateral vessels to develop and myocardial preconditioning.

Limitations: Coronary angiographic studies on the progression of coronary artery disease are subject to a methodological bias caused by the selection of patients who undergo repeat coronary arteriographic examination. An AMI event, however, offers a unique opportunity to study the progression of coronary disease over a period of time in patients who undergo 2 coronary angiograms, the first for diagnostic purposes and the second for therapeutic purposes, independently of their clinical symptoms between the two examinations.

Intermittent spontaneous reperfusion and the effects of aspirin and heparin on infarct vessel patency are difficult to assess. In addition, silent reocclusion may be undetected in some patients and may have an effect on the final infarct size. Finally, because only 43 patients were examined, the study lacks the statistical power of a large trial. Despite this limitation, these data emphasize the importance of preinfarction stenosis. Ongoing investigations directed toward understanding the development of collateral circulation may ultimately help to limit infarct size.

Conclusions: Deterioration of LV wall motion in patients with severe stenosis in an initial stenosis is milder than in AMI that developed from a mild degree of stenosis.

\section{REFERENCES}

1. Kennedy JW, Ritchie JL, Davis KB, Fritz JK. Western Washington randomized trial of intracoronary streptokinase in acute myocardial infarction. N Engl J Med 1983; 309: 1477-82.

2. Ambrose JA, Tannenbaum MA, Alexopoulos D, et al. Angiographic progression of coronary artery disease and the development of myocardial infarction. J Am Coll Cardiol 1988; 12: 56-62.

3. Visser RF, van der Werf T, Ascoop CA, Bruschke AV. The influence of anatomic evolution of coronary artery disease on left ventricular contraction: an angiographic follow-up study of 300 nonoperated patients. Am Heart J 1986; 112: 963-72.

4. Bocchi EA, Moreira LF, de Moraes AV, et al. Effects of dynamic cardiomyoplasty on regional wall motion ejection fraction, and geometry of left ventricle. Circulation 1992; 86: II231-5.

5. Cohen M, Rentrop KP. Limitation of myocardial ischemia by collateral circulation during sudden controlled coronary artery occlusion in human subjects: a prospective study. Circulation 1986; 74: 469-76.

6. Anzai T, Yoshikawa T, Asakura Y, et al. Preinfarction angina as a major predictor of left ventricular function and long-term prognosis after a first Q wave myocardial infarction. J Am Coll Cardiol 1995; 26: 319-27.

7. Kodama K, Kusuoka H, Sakai A, et al. Collateral channels that develop after an acute myocardial infarction prevent subsequent left ventricular dilation. J Am Coll Cardiol 1996; 27: 1133-9.

8. Juillière $\mathrm{Y}$, Danchin $\mathrm{N}$, Grentzinger A, et al. Role of previous angina pectoris and collateral flow to preserve left ventricular function in the presence or absence of myocardial infarction in isolated total occlusion of the left anterior descending coronary artery. Am J Cardiol 1990; 65: 277-81.

9. Marzoll U, Kleiman NS, Dunn JK, et al. Factors determining improvement in left ventricular function after reperfusion therapy for acute myocardial infarction: primacy of baseline ejection fraction. J Am Coll Cardiol 1991; 17: 613-20.

10. Monin JL, Garot J, Scherrer-Crosbie M, et al. Prediction of functional recovery of viable myocardium after delayed revascularization in postinfarction patients: accuracy of dobutamine stress echocardiography and influence of long-term vessel patency. J Am Coll Cardiol 1999; 34: 1012-9.

11. Hasche ET, Fernandes C, Freedman SB, Jeremy RW. Relation between ischemia time, infarct size, and left ventricular function in humans. Circulation 1995; 92: 710-9. 